\title{
Long non-coding RNAs in ovarian granulosa cells
}

\author{
Jiajie $\mathrm{Tu}^{1,2^{*}}, \mathrm{Yu} \mathrm{Chen}^{1+}$, Zhe Li ${ }^{1}$, Huan Yang ${ }^{1}$, He Chen ${ }^{1}$ and Zhiying Yu ${ }^{1 *}$ (D)
}

\begin{abstract}
Granulosa cells (GCs) are somatic cells surrounding oocytes within follicles and are essential for folliculogenesis. Pathological changes in GCs are found in several ovarian disorders. Recent reports have indicated that long noncoding RNAs (IncRNAs), which modulate gene expression via multiple mechanisms, are key regulators of the normal development of GCs, follicles, and ovaries. In addition, accumulating evidence has suggested that IncRNAs can be utilized as biomarkers for the diagnosis and prognosis of GC-related diseases, such as polycystic ovary syndrome (PCOS) and premature ovarian insufficiency (POI). Therefore, IncRNAs not only play a role in GCs that are involved in normal folliculogenesis, but they may also be considered as potential candidate biomarkers and therapeutic targets in GCs under pathological conditions. In the future, a detailed investigation of the in vivo delivery or targeting of IncRNAs and large-cohort-validation of the clinical applicability of IncRNAs is required.
\end{abstract}

Keywords: GCs, IncRNA, Folliculogenesis, PCOS, POI

\section{Introduction}

Granulosa cells (GCs) are somatic cells of the sex cord [1], which are associated with the development of oocytes in mammalian ovaries [2, 3]. In addition, GCs are also implicated in various ovary-related diseases, including polycystic ovary syndrome (PCOS) [4], premature ovarian insufficiency (POI, also referred to as premature ovarian failure [POF]) [5], ovarian hyperstimulation syndrome (OHSS) [6], and GCs tumor (GCT) [7]. Previous studies have shown that genetic factors are involved in the development of these diseases [8-11]. Recent evidence has shown that several non-coding RNAs also affect these female reproductive system dysfunctions.

Development of sequencing technology has led to indepth genome and transcriptome analysis, which showed that over $85 \%$ of the human genome is transcribed [12].

\footnotetext{
*Correspondence: tujiajie@ahmu.edu.cn; lizheyzy@163.com

${ }^{\dagger}$ Jiajie Tu and Yu Chen contributed equally to this work.

'Department of Gynecology, The First Affiliated Hospital of Shenzhen

University, Health Science Center, Shenzhen Second People's Hospital, 3002

Sungang West Road, Futian District, Shenzhen 518000, Guangdong province, China

Full list of author information is available at the end of the article
}

However, the amount of protein products from RNA transcripts is very low compared to the overall number of transcripts, indicating that most RNA transcripts are non-coding. Such a large number of transcripts of noncoding RNAs (ncRNAs) suggests that ncRNAs play a more important and diverse role in biological processes than initially expected $[13,14]$.

NcRNAs can be roughly divided into two groups: a group of short RNAs with a length less than 200 nucleotides long, such as microRNAs (miRNAs), small interfering RNA (siRNA), and piwi RNA (piRNA). The other category is long ncRNAs (lncRNAs) with a length longer than 200 nucleotides. Due to the lack of protein-coding capability, non-coding transcripts have been known as "junk DNA" or "transcriptional noise" for the past few decades [15]. However, many recent lncRNA-based studies have proved that lncRNAs have several functions and act via multiple regulatory mechanisms, including decoy, enhancer RNA, scaffold, guider, microRNA sponging and short peptides [16, 17]. Long non-coding RNAs are classified based on their specific characteristics and their position relative to the host or adjacent protein-

(c) The Author(s). 2020 Open Access This article is licensed under a Creative Commons Attribution 4.0 International License, which permits use, sharing, adaptation, distribution and reproduction in any medium or format, as long as you give appropriate credit to the original author(s) and the source, provide a link to the Creative Commons licence, and indicate if changes were made. The images or other third party material in this article are included in the article's Creative Commons licence, unless indicated otherwise in a credit line to the material. If material is not included in the article's Creative Commons licence and your intended use is not permitted by statutory regulation or exceeds the permitted use, you will need to obtain permission directly from the copyright holder. To view a copy of this licence, visit http://creativecommons.org/licenses/by/4.0/. The Creative Commons Public Domain Dedication waiver (http://creativecommons.org/publicdomain/zero/1.0/) applies to the data made available in this article, unless otherwise stated in a credit line to the data. 
coding gene. Location-based classification of lncRNAs assigns them into categories such as antisense RNAs, long intergenic non-coding RNAs (lincRNAs), sense overlapping transcripts, sense intronic transcripts, and processed transcripts [18]. Characteristic-based classification, on the other hand, assigns them to categories such as lncRNA-activating (lncRNA-a) genes, pseudogenes, telomere-associated non-coding RNAs (TERRAs), transcribed ultraconserved regions (T-UCRs), enhancer RNAs (eRNAs), and circular RNAs [19-21]. Compared with the multitude of studies performed on miRNAs and protein-coding genes, we are still at a relatively early stage of investigating, naming, classifying, and identifying lncRNAs.

Moreover, emerging studies have demonstrated that some lncRNAs affect the function of ovarian GCs and are thereby involved in both physiological conditions and pathological processes, such as human oocyte maturation, fertilization, embryo development, tumorigenesis, [22] and ovarian failure [23, 24]. These studies have suggested that GC-specific lncRNAs could be considered as candidate diagnostic or prognostic markers, as well as treatment targets for various ovarian diseases [25-27]. In this review, we summarize the roles of IncRNAs in healthy and dysfunctional GCs and discuss the potential utilization of IncRNAs as diagnostic markers or treatment targets in clinical conditions.

\section{The role of IncRNAs in GCs under physiological condition}

High-throughput studies of IncRNAs in normal GCs

Long non-coding RNAs are extensively transcribed and play a role in a variety of biological functions in the human genome. Communication between GCs and oocytes is essential for oogenesis [28]. Studying lncRNAs in GCs is important for a better understanding of the mechanisms of follicular maturation, fertilization, and embryo selection.

Yerushalmi et al. reported that compact GCs (CGCs) from germinal vesicle cumulus oocyte complexes (COCs) were isolated from patients undergoing in vitro maturation (IVM). Expanded GCs (EGCs) from metaphase 2 COC were isolated from patients undergoing in vitro fertilization (IVF)/intracytoplasmic sperm injection (ICSI). Global transcriptome profiles of CGCs and EGCs were compared, and 89 lncRNAs with significantly altered expressions were identified. Among them, 12 lncRNAs were encoded within introns of genes that were associated with GC development, suggesting a potential involvement of lncRNAs in cumulus expansion and oocyte maturation. Yerushalmi et al. also generated a library of genes regulated during cumulus expansion and oocyte maturation processes using RNA sequencing. Analysis of these genes led to the identification of novel lncRNAs that are potentially involved in COC maturation and cumulus expansion, which might boost our understanding of the process of oocyte maturation and ultimately improve the IVM efficiency [29].

Using microarray analysis, another study compared the expression profiles of IncRNAs in GCs from three pairs of mature oocytes that gave rise to high-quality embryos and poor-quality embryos. A total of 20,563 lncRNAs were identified in GCs. Among them, one hundred and twenty-four IncRNAs were upregulated and five hundred and nine lncRNAs were downregulated in GCs from poor-quality embryos when compared to GCs from high-quality embryos. In addition, Gene ontology (GO) was used to analyze these significantly differential expressed lncRNAs. It is believed that GC-lncRNAs may exert their functions via interactions with coding transcripts and proteins in the development of oocyte and early embryos. Therefore, lncRNAs in GCs may contribute to the process of oogenesis [30].

Another interesting study by Macaulay et al. [31] showed that GCs contribute to the maternal reserves by actively transferring a series of intermediate factors, including lncRNAs, to oocytes. This unexpected exogenous trafficking to the maternal storage offers a new perspective on the determinants of female fertility. However, the exact molecular mechanism of this communication between GCs and oocyte is yet to be revealed. The specific role lncRNAs, in this transferring, warrants further investigation.

Published sequencing data [32, 33] were used to identify the abundant lncRNAs in metaphase II (MII) oocytes and surrounding GCs. The function of the identified lncRNAs was predicted by the expression network of lncRNA-mRNA. Bioinformatic analysis showed that several lncRNAs (NEAT1, MALAT1, ANXA2P2, MEG3, IL6STP1 and VIM-AS1) in GCs are involved in apoptosis and extracellular matrix-related functions, indicating that lncRNAs in GCs were essential for oocytes growth. This study of lncRNAs in human MII oocytes and GCs provided potential biomarkers for the identification of embryos with high development potential [34]. The above-mentioned studies indicated that lncRNAs expression is significantly altered in GCs [29, 30, 34]. These IncRNAs may play an essential role in female reproduction by directly cross-talking with oocytes [31].

\section{Individual studies of IncRNAs in normal GCs LnCRNA-LET}

The endogenous expression of LncRNA-LET in KGN cells is quite low. Overexpression of this lncRNA in KGN cells repressed proliferation and migration and induced apoptosis. LncRNA-LET also repressed KGN cell epithelial-mesenchymal transition (EMT) by inducing Ecadherin and reducing $\mathrm{N}$-cadherin and vimentin expression. An essential EMT-associated factor, tissue inhibitor 
of metalloproteinases 2 (TIMP2), was directly induced by lncRNA-LET. In addition, TIMP2 overexpression generally mimicked the lncRNA-LET function in KGN cells, validating the idea of TIMP2 being downstream target of lncRNA-LET. Moreover, lncRNA-LET and TIMP2 activated the Wnt/ $\beta$-catenin and Notch pathways in KGN cells. Overall, lncRNA-LET repressed proliferation, migration and EMT, and promoted apoptosis of KGN cells by activating TIMP2 and inducing the Wnt/B-catenin/Notch pathways [35].

\section{LncRNA-Amhr2}

Anti-Müllerian hormone (AMH) is mainly produced and secreted from GCs and is essential for repressing Mullerian duct development [36]. The long non-coding RNA, IncRNA-Amhr2, which is transcribed from the upstream region of the AMH receptor type 2 (Amhr2), was identified in GCs. Amhr2 expression was inhibited in lncRNA-Amhr2 knockdown mouse primary GCs (the isolated primary mouse GCs are mixed cumulus GCs and mural GCs). In addition, reporter assay results showed that lncRNA-Amhr2 activation induced Amhr2 promoter activity. This direct transcriptional regulation was further validated in a mouse GCs line (OV3121 cells). This study suggested that IncRNA-Amhr2 activated the transcription of its neighboring gene Amhr2 in GCs, demonstrating a novel mechanism of Amhr2 regulation in the female reproductive system [37].

\section{LncPrep96kb}

Prolyl oligopeptidase (POP) is a serine endopeptidase that is involved in progesterone secretion [38]. To further investigate the regulation of Prolyl oligopeptidase (POP) in GCs, the genomic loci of POP and six neighboring lncRNAs were studied. Since there are two different transcriptional start sites (TSS), the one adjacent to lncRNA-lncPrepp96kb was transcribed as two isoforms. This lncRNA induced POP in primary ovarian GCs (the isolated primary mouse GCs are mixed cumulus GCs and mural GCs), and both lncPrepp96kb and POP were up-regulated in the hormone-treated ovaries [39].

\section{LnCRNA-AK124742}

LncRNA-AK124742 was identified as an antisense of proteasome 26S subunit, non-ATPase, 6 (PSMD6). A positive association was found between AK124742 and PSMD6 in cumulus GCs. Up-regulations of AK124742 and PSMD6 were observed in high-quality embryos (HCCs) compared to poor-quality embryos (PCCs). The relative expressions of AK124742 and PSMD6 were upregulated in the pregnancy group compared to those in the non-pregnancy group. AK124742-PSMD6 is a potential lncRNA-mRNA biomarker in cumulus GCs involved in embryo selection [24].

\section{LnCRNA-HAS2-AS1}

Cumulus expansion is a luteinizing hormone (LH)-mediated ovulatory process. Hyaluronan synthase 2 (HAS2) affects the synthesis of hyaluronic acid (HA), a major component of cumulus expansion. LncRNA HAS2 antisense RNA 1 (HAS2-AS1) expression was low in immature GCs and up-regulated in mature GCs. HAS2-AS1 inhibition caused the suppression of HAS2 and repressed the migration of cumulus GCs, suggesting that HAS2-AS1 is an LH-targeted gene that modulates cumulus expansion and migration by increasing HAS2 expression [40].

To summarize, these studies in the above section focused on the roles of five individual lncRNAs in normal GCs, and their findings suggested that IncRNAs could affect the GCs and ovaries via different mechanisms. Although the role of IncRNAs in GCs have not been well-established under physiological conditions, the aforementioned reports show that they play an essential role in GCs. Specifically, IncRNAs (including Amhr2, lncPrep96kb and HAS2-AS1) regulate host or adjacent gene function in GCs. In addition, lncRNAs (such as AK124742) could be potential biomarkers of embryo selection. Individual lncRNA (LncRNA-LET) also affects the functions of GCs (proliferation, migration, and EMT) by affecting traditional signaling pathways (Wnt signaling pathway). The role of individual lncRNA in normal GCs was summarized in Fig. 1.

\section{LncRNAs in PCOS GCs}

\section{High-throughput studies of IncRNAs in PCOS GCs}

Polycystic ovary syndrome (PCOS) is the most common cause of anovulatory infertility in women, mainly manifesting polycystic ovary and hyperandrogenism. The potential role of lncRNAs in the pathogenesis of PCOS was studied in human primary GCs and a GCs cell line, human granulosa-like tumor cell line (KGN). Microarrays were performed to compare "IncRNAome" in primary GCs obtained from seven PCOS patients and seven control women. The primary human GCs were mainly cumulus GCs. In total, 862 lncRNAs and 998 mRNAs were differentially expressed in the PCOS GCs. Real-time reverse transcription PCR (qRT-PCR) was used to validate lncRNA HCG26 expression in GCs obtained from 53 PCOS patients and 50 controls. HCG26 expression was upregulated in PCOS patients and positively correlated with follicle number at a mature stage. HCG26 knockdown in KGN cells inhibited cell proliferation and cell-cycle progression. In addition, aromatase gene (CYP19A1) expression and estradiol production were also increased in HCG26-knockdown KGN cells. This study was the first to report the lncRNA profiles in GCs obtained from PCOS patients, and suggested that dysregulated lncRNAs might play vital roles in GCs proliferation and steroidogenesis [41]. 


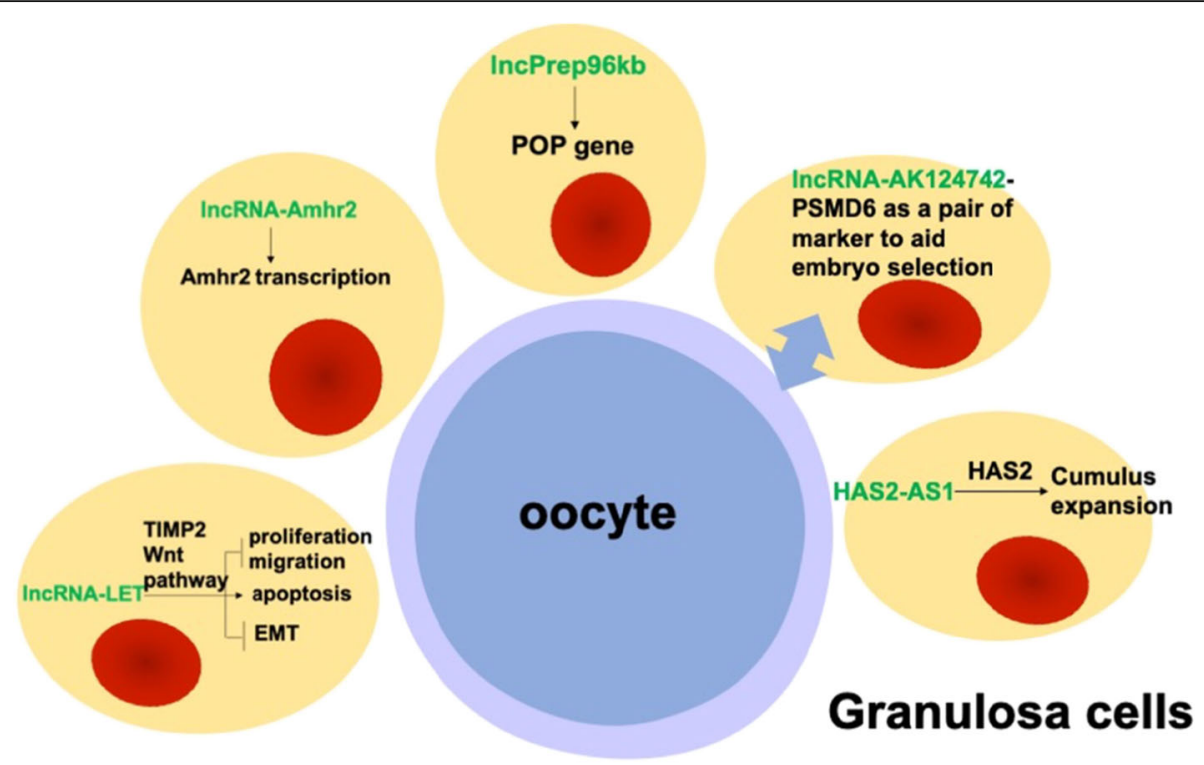

Fig. 1 The role of individual IncRNAs in normal granulosa cells (GCs). IncRNA-LET repressed proliferation, migration, epithelial-mesenchymal transition (EMT) and promoted apoptosis in a human GCs line KGN by activating TIMP2 and inducing the Wnt/3-catenin/Notch pathways; IncRNA-Amhr2 activated the transcription of its neighboring gene Amhr2 in primary mural and cumulus GCs and in a mouse GC line OV3121 cells; IncRNA-IncPrepp96kb induced POP in primary ovarian mural and cumulus GCs; AK124742-PSMD6 is a potential IncRNA-mRNA biomarker in cumulus GCs for embryo selection; HAS2-AS1 caused activation of HAS2 and induced cumulus GCs migration

Another high-throughput study showed that 623 lncRNAs and 260 mRNAs were significantly altered and these differences could be used to discriminate GCs from PCOS patients and normal controls. Interestingly, some differentially expressed lncRNAs that are transcribed from chromosome 2 could act as enhancers, regulating neighboring coding genes. Therefore, we compared all differentially expressed lncRNAs that are transcribed from chromosome 2 and enhancer-like lncRNAs that were differently expressed in PCOS GCs. Fourteen overlapping lncRNAs were found among these two lncRNA sets (full list in supplementary Table 1). The relationship between these enhancer-like lncRNAs and their neighboring coding genes in GCs requires further investigation. In addition, among all the significantly up- or down-regulated lncRNAs and mRNAs, 43 lncRNAs and 29 mRNAs were closely correlated to construct "coding and non-coding gene networks." Most IncRNA-mRNA pairs correlated positively. These results showed that lncRNAs are aberrantly expressed in GCs of PCOS patients. Dysfunction of these IncRNAs might account for the occurrence of PCOS and affect oocyte development [42].

According to the guidelines of both the Rotterdam or National Institutes of Health, hyperandrogenism is one of the most important diagnostic criteria of PCOS [43]. Hyperandrogenism, abnormal follicular development and ovulation largely result from GCs dysfunction [44]. LncRNAs were profiled in GCs that isolated from 4 women with PCOS with hyperandrogenism (PCOS-T) or without hyperandrogenism (PCOS-N) and healthy controls. Compared to the control group and PCOS-N, 3000 and 1030 lncRNAs were significantly altered in PCOS-T GCs ( $\geq$ two-fold change). In addition, a total of 1361 differentially expressed lncRNAs were detected in PCOS-N compared to the control group. Corticotropin releasing hormone binding protein (CRHBP) was the largest up-regulated lncRNA in PCOS-T compared to either PCOS-N or control. GO and KEGG pathway analysis suggested that CRHBP plays an essential role in mitochondrial function by interacting with transcription factors such as YY1 and SIX5 in PCOS-T GCs. This group of lncRNAs, represented by CHBRP, may play a key role in the steroid genesis and metabolism in PCOS [45].

\section{Indvidual studies of IncRNAs in PCOS GCs LnCRNA-SRA}

The levels of IncRNA steroid receptor RNA activator (SRA) had increased in the peripheral blood of PCOS patients. SRA overexpression promoted GCs (the isolated primary mouse GCs are mixed cumulus GCs and mural GCs) growth and regulated the cell cycle by elevating several cell-cycle associated proteins, including Cyclin B, Cyclin E, and Cyclin D1. SRA also inhibited cell apoptosis by increasing the ratio of bcl-2:bax and repressing the levels of cleaved-caspase 3 and cleavedPoly (ADP-Ribose) polymerase (PARP). In addition, SRA elevated the production of estradiol and progesterone, 
and that of two key enzymes, CYP19A1 and CYP11A1, in GCs, suggesting that SRA is a potential risk factor for PCOS [46]. A follow-up study on SRA from the same group further showed that SRA promoted ovary injury and the production of angiogenic factors in a mouse model of PCOS. Moreover, SRA increased the production of pro-inflammatory cytokines and stimulated NF$\kappa B$ nuclear translocation in primary GCs obtained from PCOS mice. This study validated the important role of SRA in the development of PCOS [47].

\section{LnCRNA-PWRN2}

Prader-Willi region non-protein coding RNA 2 (PWRN2) expression was associated with oocyte nuclear maturation in PCOS patients. In cumulus GCs, PWRN2 regulates $176 \operatorname{lncRNAs}$ and 131 mRNAs. Based on these microarray data, a PWRN2-miR-92b-3p-transmembrane protein 120B (TMEM120B) competing endogenous RNA (ceRNA) network was established. This network was further validated by a luciferase report assay that revealed that miR-92b-3p directly binds to PWRN2 and TMEM120B. Therefore, PWRN2 is involved in oocyte nuclear maturation in PCOS patients by acting as a ceRNA [48]. Interestingly, PWRN2 was also identified as a significantly up-regulated lncRNA in genomic screening of another group of PCOS patients [42], validating that PWRN2 is indeed an essential lncRNA in a larger cohort of PCOS patients.

\section{LnCRNA-BANCR}

BRAF-activated non-protein coding RNA (BANCR) is a well-studied lncRNA that plays pivotal roles in various malignancies, such as melanoma [49] and endometrial cancer [50]. BANCR acts as an "onco-lncRNA" via interactions with ERK/MAPK pathways. BANCR expression was significantly higher in cumulus GCs obtained from PCOS patients. Insulin treatment up-regulated BANCR expression in primary cumulus GCs and in the GCs line, KGN. BANCR overexpression repressed KGN cell proliferation and induced KGN cell apoptosis by promoting two pro-apoptotic markers, Bax and p53, suggesting that BANCR participates in PCOS by promoting cumulus GC apoptosis [51].

\section{LncRNA- LINC-01572:28}

In human primary cumulus GCs obtained from PCOS patients a negative association was found between LncRNA LINC-01572:28/p27 protein and PCNA. Also, there was a positive correlation between LINC-01572:28 expression and testosterone secretion from primary $\mathrm{cu}-$ mulus GCs. Furthermore, LINC-01572:28 inhibited cumulus GCs proliferation and prevented G1/S transition, which were partially reversed by p27 knockdown. This indicated that LINC-01572:28 suppressed cumulus GCs proliferation and cell cycle progression by reducing the degradation of p27 protein via competitive binding to Sphase kinase associated protein 2 (SKP2)-a key component of the SCF-SKP2 ubiquitin-ligase complex, which mediates cyclin E/CDK2-dependent ubiquitination and degradation of p27 [52].

\section{LnCRNA-HUPCOS}

Androgen overdose is a key feature of PCOS. Researchers used microarrays to measure the profile of the abnormally expressed lncRNAs in GCs from 8 PCOS patients. A very highly expressed lncRNA in PCOS GCs was named as HUPCOS, which was positively correlated with follicle testosterone in PCOS patients. HUPCOS knockdown increased aromatase expression, which also promoted the conversion of androgens to estrogen. In addition, RNA-binding protein with multiple splicing RBPMS was identified as a direct binding protein of HUPCOS. This study showed that a novel IncRNA, HUPCOS, mediated androgen excess in the follicular fluid of PCOS patients by inhibiting aromatase expression via interaction with RBPMS [53].

\section{LnCRNA-PVT1}

Liu et al. studied the role of lncRNA-PVT1/miR-17-5p/ PTEN axis in PCOS ovarian GCs. Binding between PVT1 and miR-17-5p and the targeting between miR17-5p and PTEN are determined by bioinformatics analysis, luciferase activity assay, RNA-induced silencing complex assay, and RNA pull-assay. PVT1 and PTEN were both highly expressed in PCOS ovarian GCs and follicular fluid, whereas miR-17-5p was not. MiR-17-5p overexpression and PVT1 knockdown could slow apoptosis and promote the cloning formation and proliferation of ovarian GCs in PCOS. In addition, PVT1 overexpression and miR-17-5p inhibition reversed these results. This study showed that both PVT1 downregulation and miR-17-5p up-regulation lead to PTEN inhibition, which promoting proliferation and repressing apoptosis of ovarian GCs in PCOS [54].

\section{LnCRNA-TUG1}

LncRNA TUG1 was significantly high in PCOS GCs and was associated with the antral follicle count. TUG1 was mainly expressed in GC nucleus. TUG1 knockdown in KGN cells inhibited proliferation and promoted apoptosis of GCs. TUG1 knockdown led to increased expression of bax, bak, cleaved caspase-3, caspase-9, cleaved caspase-9, LC3B and p-ERK proteins and decreased expression of bcl-2 and $\mathrm{p} 62$ proteins. In addition, using the ERK/MAPK pathway inhibitor U0126, the TUG1 knockdown-induced change in KGN cells was partially restored. Therefore, TUG1 was significantly higher in the PCOS group than that in the control group, TUG1 
may inhibit cell apoptosis and autophagy in GCs through ERK/MAPK pathway inhibition and contribute to excess antral follicles. TUG1 has potential diagnostic value in PCOS [55].

Taken together, these studies suggested that GCspecific lncRNAs are involved in PCOS, which paves a novel pathway for better understanding of the molecular mechanisms underlying this reproductive disease. However, these high-throughput experiments of lncRNA only identified GC-specific IncRNAs at the transcriptome level (Table 1). To identify potential specific diagnostic markers and therapeutic targets, we need to study the specific function of individual lncRNAs in GCs from patients with PCOS. Until now, nine individual lncRNAs have been identified in PCOS GCs (Fig. 2), most of which are involved in two main cellular functions of GCs: proliferation and hormone production. Synergistic effects between lncRNAs with similar functions should be further investigated in the future.

\section{The role IncRNAs in POI GCs}

Premature ovarian insufficiency (POI, also referred to as premature ovarian failure [POF]) is a common cause of female infertility. GC apoptosis is one important mechanism underlying this decline in ovarian function [56]. Cyclophosphamide treatment was found to repress GCs growth and lead to ovarian atrophy. Northern blot results demonstrated that the intensity of the lncRNAMeg3 hybridization signal was up-regulated in cyclophosphamide-treated GCs (the isolated primary mouse GCs are mixed cumulus GCs and mural GCs). Expressions of apoptotic markers, including p53, p66Shc, p16, and cleaved caspase-3, were all decreased in lncRNA-Meg3-knockdown GCs compared to control. Thus, cyclophosphamide deferred GCs proliferation and promoted POF by inducing lncRNA-Meg3 [23].

Wang et al. found lncRNA HCP5 decreased in GCs that isolated from patients with biochemical POI (patients with normal menstrual cycle and increased gonadotropin). HCP5 stabilized the interaction between YB1 and ILF2, mediating the YB1 transfer into GC nucleus. HCP5 knockdown affected this transfer and reduced its binding to to the promoter region of MSH5 gene and repressing its transcription. Reduced HCP5 expression in GCs of biochemical POI patients contributed to the generation of dysfunctional GCs by modulating MSH5 transcription via the interaction with YB1. This study showed a novel epigenetic mechanism for POI pathogenesis [57].

To investigate whether IncRNA-FMR6 is involved in the development of fragile $\mathrm{X}$-associated premature ovarian insufficiency (FXPOI), 22 consecutive fragile $\mathrm{X}$ mental

Table 1 LncRNA expression profiling in granulosa cells (GCs)

\begin{tabular}{|c|c|c|c|c|c|}
\hline No. & platform & sample & GEO No. & $\begin{array}{l}\text { Upregulated/downregulated } \\
\text { IncRNAs }\end{array}$ & Ref \\
\hline 1 & $\begin{array}{l}\text { Illumina Cluster Station for } \\
\text { cDNA library preparation } \\
\text { and Genome } \\
\text { Analyzer:HiSeq } 2000 \\
\text { seqencer }\end{array}$ & $\begin{array}{l}2 \text { CCGV patients and } 3 \\
\text { CCM2 patients (control) }\end{array}$ & GSE50174 & 28/61 IncRNAs & 29 \\
\hline 2 & $\begin{array}{l}\text { Arraystar Human } 8 \times 60 \mathrm{~K} \\
\text { IncRNA Microarray V2.0 }\end{array}$ & $\begin{array}{l}3 \text { pairs of GC from mature } \\
\text { oocytes that in high- and } \\
\text { poor-quality embryos }\end{array}$ & None & 124/509 IncRNAs & 30 \\
\hline 3 & Illumina Hiseq 2000 & $\begin{array}{l}10 \text { MII oocytes, } 10 \text { GCs } \\
\text { samples and } 97 \text { pre- } \\
\text { implantation embryos }\end{array}$ & $\begin{array}{l}\text { GSE26552 } \\
\text { GSE44183 }\end{array}$ & $6236 / 2809$ IncRNAs & 34 \\
\hline 4 & $\begin{array}{l}\text { Arraystra Human } \\
\text { LncRNA Microarray } \\
\text { V3.0 }\end{array}$ & $\begin{array}{l}7 \text { PCOS patients and } 7 \\
\text { matched women }\end{array}$ & GSE95728 & $602 / 170$ IncRNAs & 41 \\
\hline 5 & $\begin{array}{l}\text { Agilent-038314 CBC } \\
\text { Homo sapiens IncRNA + } \\
\text { mRNA microarray V2.0 }\end{array}$ & $\begin{array}{l}5 \text { PCOS patients and } 5 \\
\text { matched women }\end{array}$ & GSE65746 & $620 / 3$ IncRNAs & 42 \\
\hline 6 & $\begin{array}{l}\text { Agilent Human IncRNA } \\
(4 * 180 \text { K, Design ID: } \\
\text { 062918) array }\end{array}$ & $\begin{array}{l}4 \text { PCOS women with } \\
\text { hyperandrogenism(PCOS-T), } 4 \\
\text { PCOS women without } \\
\text { hyperandrogenism(PCOS-N) } \\
\text { and } 4 \text { control participants }\end{array}$ & GSE106724 & $\begin{array}{l}\text { PCOS-T vs PCOS-N: } 445 / 585 \text { IncRNAs } \\
\text { PCOS-T vs control: } 971 / 2029 \text { IncRNAs } \\
\text { PCOS-N vs control: } 1361 \text { differentially } \\
\text { expressed IncRNAs }\end{array}$ & 45 \\
\hline 7 & $\begin{array}{l}\text { Illumina HiSeq }{ }^{\mathrm{TM}} \times 10 \\
\text { sequencer }\end{array}$ & $\begin{array}{l}3 \text { patients with OHSS high } \\
\text { risk and } 3 \text { patients with } \\
\text { OHSS low-risk }\end{array}$ & None & 205/277 IncRNAs & 59 \\
\hline 8 & Illumina HiSeq 2500 & $\begin{array}{l}\text { TCDD-treated AVG-16 } \\
\text { porcine GC }\end{array}$ & None & $\begin{array}{l}22 \text { IncRNAs differentially expressed } \\
\text { in GC treated with TCDD for } 3,12 \text { or } \\
24 \mathrm{~h} \text {. }\end{array}$ & 62 \\
\hline
\end{tabular}




\section{Immature follicle from PCOS patients}

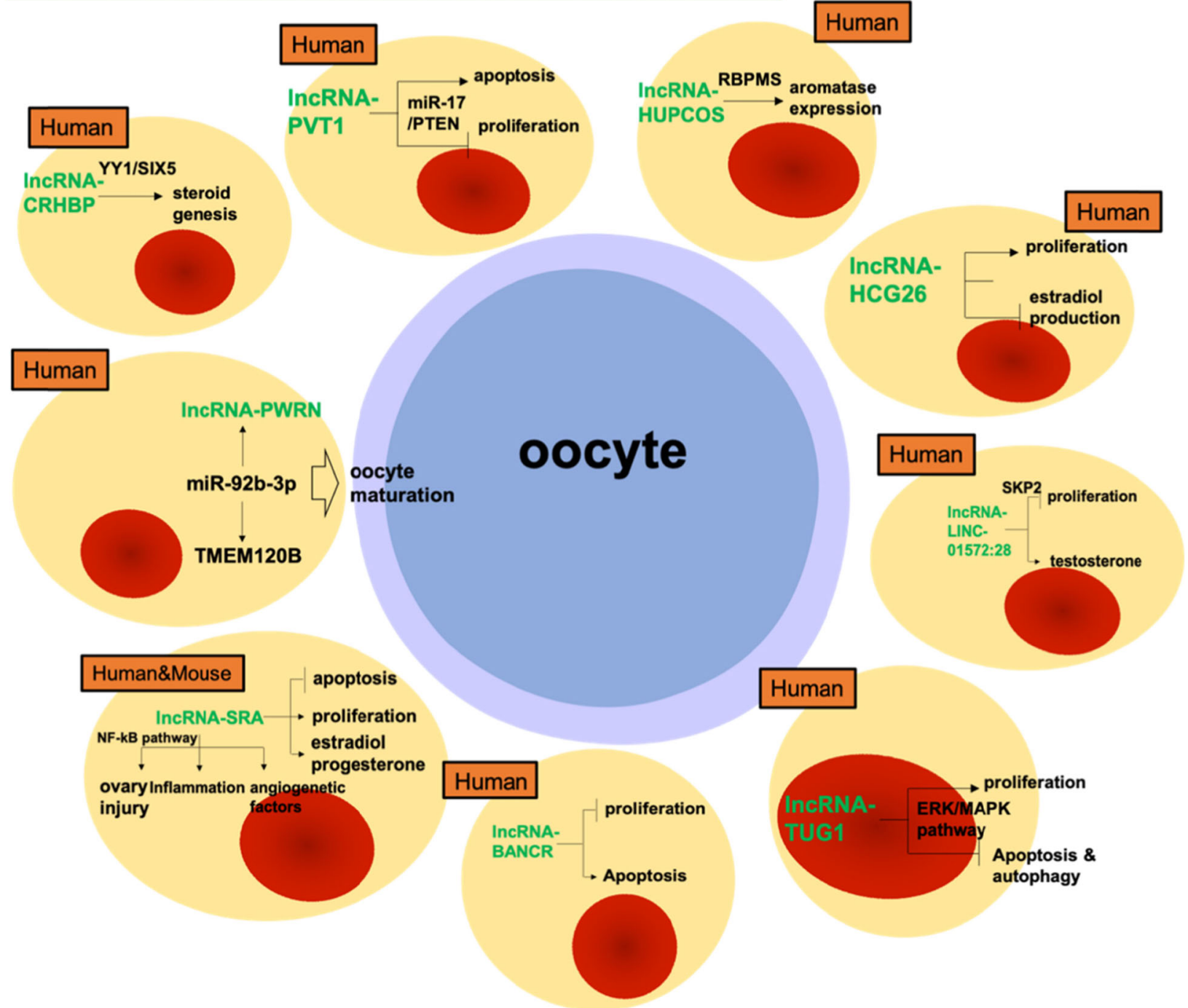

Fig. 2 IncRNAs in GCs obtained from polycystic ovary syndrome (PCOS) patients. SRA is a potential risk factor for PCOS as it affects several functions of mural and cumulus GCs, including proliferation, apoptosis, inflammation, and production of angiogenic factors and hormones; PWNR2 is involved in oocyte nuclear maturation in PCOS by functioning as a ceRNA in cumulus GCs; BANCR participates in PCOS progression by promoting cumulus GCs apoptosis; LINC-01572:28 suppresses PCOS cumulus GCs proliferation and cell cycle progression via competitive binding to SKP2; TUG1 inhibits apoptosis and autophagy in PCOS GCs through inhibition of ERK/MAPK pathway; HUPCOS mediates androgen excess in follicular fluid of PCOS patients via interaction with RBPMS; CHBRP involves in steroid genesis and metabolism in PCOS GCs by interacting with transcription factors YY1 and SIX5; HCG26 knockdown in GCs inhibits cell proliferation induces estradiol production. PVT1 down-regulation and miR-17-5p up-regulation lead to PTEN inhibition, which promoting proliferation and repressing apoptosis of PCOS GCS

retardation 1 (FMR1) premutation carriers undergoing IVF and pre-implantation genetic diagnosis (IVF-PGD) were studied. Eleven patients undergoing IVF- intracytoplasmic sperm injection (ICSI) were recruited as a control group and their cumulus GCs were isolated after oocyte retrieval. In FMR1 premutation carriers, there was a nonlinear association between the number of CGG repeats and the transcriptional level of lncRNA-FMR6. A negative linear correlation was observed between the number of oocytes retrieved and lncRNA-FMR6 expression in cumulus GCs, indicating that the RNA toxic gain-of-function is a potential pathophysiological mechanism of FXPOI [58].

Ovarian reserves depend primarily on the number and quality of oocytes. Initially, insufficient or accelerated depletion of the primordial follicle pool will result in ovary insufficiency. It has been determined that mammalian GCs significantly contribute to the development of follicles $(39,40)$. In addition, reduced GCs induced follicle 
atresia, which eventually lead to POI $(18,19)$. So far, the main research field of POI pathogenesis was proteincoding genes (4-6). However, the causing-gene mutations can only explain $\sim 15 \%$ of POI cases $(35,36)$. There is currently a lack of research on the role of individual lncRNA in POI GCs (only three reports until now, as depicted in Fig. 3). In the future, IncRNAs sequencing in POI GCs should be performed to better understand the role of lncRNAs in POI from a panoramic view, which will explore unidentified essential individual lncRNA role in POI.

\section{The role of IncRNAs in other GC-related diseases}

To identify differentially expressed lncRNAs in ovarian hyperstimulation syndrome (OHSS), which is characterized by enlarged ovaries and up-regulated vascular permeability, GCs were obtained from women with varying OHSS risks, for high-throughput sequencing. A total of 23,815 lncRNAs were detected and 482 of them showed differential expression: 205 lncRNAs were up-regulated and 277 lncRNAs were downregulated. Several ovarian biological processes were significantly involved in this phenomenon as demonstrated by KEGG pathway and GO analyses. Meanwhile, an lncRNA/miRNA interacting network was also established according to ceRNA regulatory mechanisms, a recent finding that adds to the complexities of miRNA-mediated gene modulation. ceRNAs are RNAs that mutually regulate other miRNAs via competitively binding the same miRNA recognition elements (MREs). In addition, expression screening identified eight novel lncRNAs (Supplementary Table 2) in GCs that were associated with risk factors for OHSS, suggesting that these lncRNAs might be potential participants in OHSS development [59].

The environmental contaminant, 2,3,7,8-tetrachlorodibenzo-p-dioxin (TCDD), causes reproductive defects, such as anovulation and disorder of follicular steroidogenesis, in several mammals, including human, mouse, rat and pig [60, 61]. In total, 1666 lncRNAs were characterized in TCDD-treated porcine GCs in vitro and 22 differentially expressed lncRNAs were identified. The potential functions of these 22 lncRNAs were predicted by analyzing their cis- and trans-regulated proteincoding genes. Two essential functional proteins of GCs, cytochrome P450 1A1 (CYP1A1) and aryl hydrocarbon receptor (AhR), were identified among the genes that were trans-regulated by the differentially expressed lncRNAs. The mRNA-lncRNA co-expression analysis showed that the TCDD-regulated lncRNAs might be involved in numerous cellular activities in GCs, such as

\section{Follicle from POI patients}

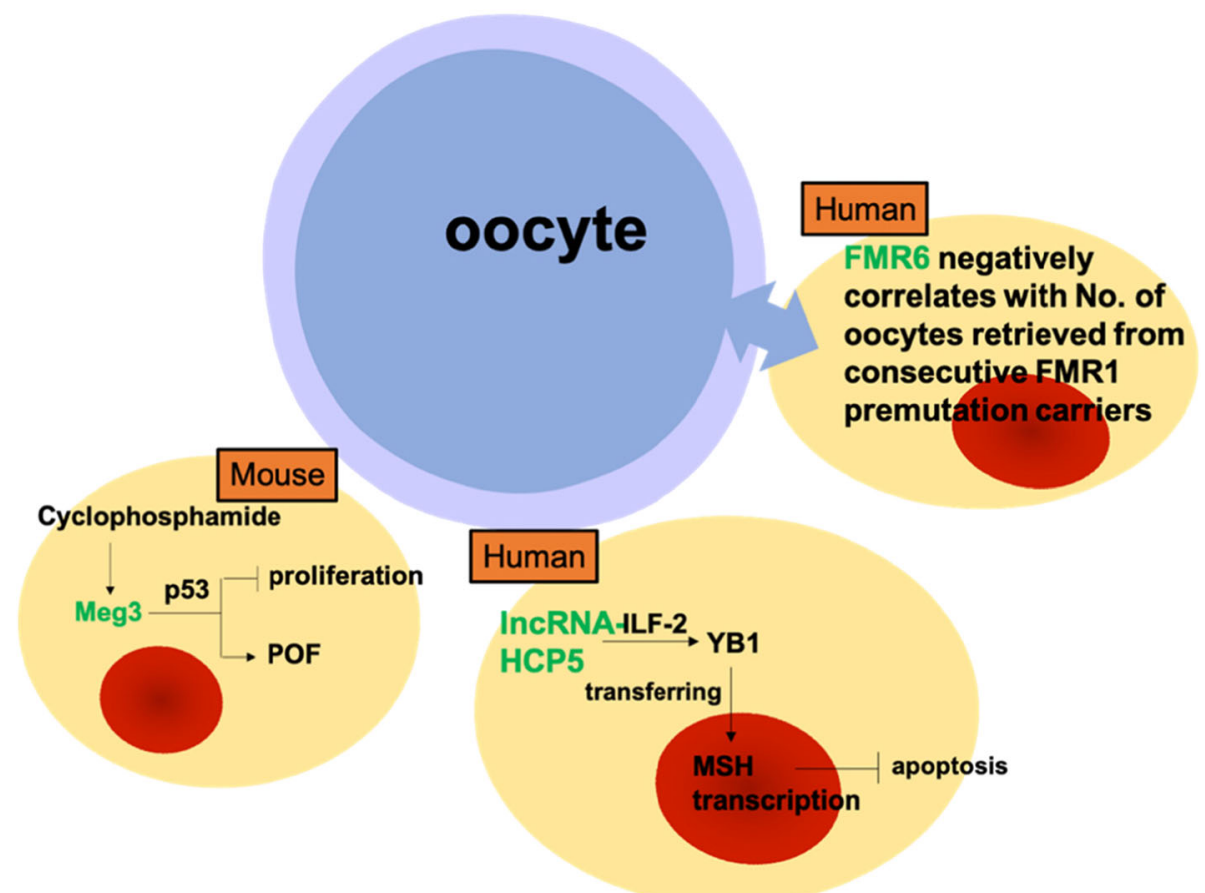

Fig. 3 LncRNAs in GCs obtained from Premature ovarian failure (POI) patients. Cyclophosphamide defers mural and cumulus GCs proliferation and promotes POF by inducing IncRNA-Meg3; FMR6 correlates negatively with the number of oocytes retrieved from consecutive FMR1 premutation carriers in cumulus GCs; HCP5 contributed to dysfunctional GCs by modulating MSH5 transcription via the interaction with YB1 
Table 2 Summary of regulatory mechanisms of IncRNAs in granulosa cells (GCS)

\begin{tabular}{|c|c|c|c|}
\hline No. & Mechanism & IncRNA & Ref \\
\hline \multirow[t]{4}{*}{1} & \multirow{4}{*}{$\begin{array}{l}\text { LncRNAs regulate classical signaling } \\
\text { pathways in GCs }\end{array}$} & IncRNA-LET induces Wnt/ $\beta$-catenin/Notch pathways & 35 \\
\hline & & IncRNA-SRA activates NF- $\mathrm{kB}$ pathway & 47 \\
\hline & & IncRNA-MALAT1 represses ERK/MARK pathway & 66 \\
\hline & & $\begin{array}{l}\text { TUG1 inhibits apoptosis and autophagy in GCs through } \\
\text { inhibition of ERK/MAPK pathway. }\end{array}$ & 55 \\
\hline \multirow[t]{3}{*}{2} & \multirow[t]{3}{*}{$\begin{array}{l}\text { LncRNAs activate the transcription via } \\
\text { activating their host genes or adjacent } \\
\text { coding genes in GCs }\end{array}$} & $\begin{array}{l}\text { IncRNA-Amhr2 activated the transcription of its neighboring } \\
\text { gene Amhr2 }\end{array}$ & 36 \\
\hline & & $\begin{array}{l}\text { IncRNA-IncPrepp96kb promotes its adjacent coding gene } \\
\text { POP }\end{array}$ & 39 \\
\hline & & HAS2 was activated by its antisense IncRNA-HAS2-AS1 & 40 \\
\hline \multirow[t]{3}{*}{3} & \multirow[t]{3}{*}{ ceRNA mechanism in GCs } & PWRN2-miR-92b-3p-TMEM120B ceRNA network & 48 \\
\hline & & LncRNA-LINC-01572:28/p27-SKP2-p27 ceRNA network & 52 \\
\hline & & PVT1-miR-17-5p-PTEN ceRNA network & 54 \\
\hline \multirow[t]{2}{*}{4} & \multirow[t]{2}{*}{ P53-associated IncRNAs } & IncRNA-BANCR & 51 \\
\hline & & LncRNA-Meg3 & \\
\hline \multirow[t]{3}{*}{5} & \multirow[t]{3}{*}{ Interaction with transcriptional factors } & $\begin{array}{l}\text { HUPCOS mediates androgen excess in follicular fluid of } \\
\text { PCOS patients via interaction with RBPMS }\end{array}$ & 53 \\
\hline & & $\begin{array}{l}\text { CHBRP involves in steroid genesis and metabolism in PCOS } \\
\text { by interacting with transcription factors YY1 and SIX5 }\end{array}$ & 45 \\
\hline & & $\begin{array}{l}\text { HCP5 contributed to dysfunctional GCs by modulating MSH5 } \\
\text { transcription via interaction with YB1. }\end{array}$ & 57 \\
\hline
\end{tabular}

cellular response to xenobiotics, proliferation, and dioxin metabolism. Therefore, these GC-specific lncRNAs may be involved in TCDD-induced reproductive defects [62].

Endometriosis and its surgical treatment have an adverse effect on the ovarian reserve and on oocyte development. Metastasis associated lung adenocarcinoma transcript 1 (MALAT1), also known as nuclear-enriched abundant transcript 2 (NEAT2), is a well-studied lncRNA that is highly evolutionarily conserved [63] and is extensively investigated as an "onco-lncRNA" in various malignancies [64, 65]. Its expression was downregulated in GCs (the isolated primary mouse GCs are mixed cumulus GCs and mural GCs) from patients with endometriosis. Moreover, MALAT1knockdown repressed GCs proliferation via the induction of the extracellular signal-regulated kinase (ERK)/mitogen-activated protein kinase (MAPK) pathway, suggesting that the MALAT1 dysfunction might have an adverse effect on the development of oocytes in endometriosis [66].

Taken together, these data reflect the important role of GC-specific lncRNAs in a series of GC-related reproductive diseases, suggesting that GC-specific lncRNAs may be used as diagnostic markers or potential therapeutic targets for these diseases. However, the underlying mechanisms have not been systematically illuminated and these studies used in vitro assays. Therefore, to elucidate the specific function and mechanism of these lncRNAs in GCs obtained from patients suffering from ovarian diseases, advanced animal models and a large number of clinical samples will be required in subsequent studies.

\section{LncRNAs as targets for diagnosis and treatment of GC-related diseases}

To investigate the potential application of lncRNAs in clinical diagnosis and therapy of GC-related diseases, researchers currently focus on the function and underlying mechanisms of lncRNAs in GCs. High-throughput technologies, such as lncRNA sequencing, have been applied to screen lncRNAs in GCs in several pathological conditions. These differentially expressed lncRNAs may be useful for the diagnosis or prognosis of GC-associated diseases. Moreover, studies of individual lncRNAs in GCs highlight the potential use of IncRNAs as therapeutic targets for GCrelated diseases. For example, encapsulated siRNA may be delivered to target specific lncRNAs for the treatment of GC-related diseases. Due to the complex spatial structure and unclear molecular regulatory mechanisms, studies of lncRNAs are still at a very early stage. Therefore, a deeper understanding of lncRNA functions in GCs will provide a 
promising foundation for a potential future use of lncRNAs in the diagnosis or treatment of GC-related diseases.

\section{Conclusion and future perspectives}

Granulosa cells are widely known for playing an essential role in both normal folliculogenesis and various ovarian disorders. LncRNAs exert their effects in GCs via multiple mechanisms. Until now, all regulatory mechanisms of lncRNA in GCs could be divided into five categories: 1) lncRNAs that regulate classical signaling pathways, such as lncRNA-LET (which induces the Wnt/ $\beta$-catenin/Notch pathways), or lncRNA-SRA (which activates the NF- $\mathrm{KB}$ pathway) and IncRNA-MALAT1 and IncRNATUG1(which represses the ERK/MARK pathway) in GCs; 2) lncRNAs that activate transcription via activating their host genes or adjacent coding genes in GCs, including lncRNA-Amhr2, which activates the transcription of its neighboring gene Amhr2, or lncRNA-lncPrepp96kb, which promotes its adjacent coding gene POP and HAS2, which was activated by its antisense IncRNA-HAS2-AS1; 3) ceRNA mechanism in GCs: PWRN2-miR-92b-3pTMEM120B ceRNA network, LncRNA-LINC-01572:28/ p27-SKP2-p27 ceRNA network and PVT1-miR-17-5pPTEN ceRNA network in GCs; 4) P53-associated lncRNAs: lncRNA-BANCR and LncRNA-Meg3; 5) transcriptional factors-associated lncRNAs: lncRNA-HUPCOS interacts with RBPMS, IncRNA-CHBRP interacts with YY1 and SIX5 and lncRNA-HCP5 interacts with YB1(Table 2). The specific functional roles, upstream regulators, and downstream effectors of these dysregulated lncRNAs in GCs are still elusive. The dysregulated expression of lncRNAs in the circulation has been used as a biomarker in several studies that allowed distinguishing patients with ovarian disorders from healthy controls; however, the clinical usage of lncRNAs for ovarian disorder diagnosis remains to be established through large-cohort validation in different populations. In the future, advanced technologies, such as single-cell sequencing, CRISPR-Cas9, genetic animal models, and subsequent clinical trials must be employed to further elucidate the mechanisms and verify the clinical potential of lncRNAs in GCs as diagnostic or therapeutic targets for ovarian disorders.

\section{Supplementary information}

Supplementary information accompanies this paper at https://doi.org/10. 1186/s13048-020-00663-2.

Additional file 1. The fourteen overlapping IncRNAs that are transcribed from chromosome 2 and enhancer-like IncRNAs that were differently expressed in PCOS GCS.

Additional file 2. The eight novel IncRNAs in GCs that were associated with risk factors for OHSS.
Authors' contributions

All authors contributed to the drafting and revising of the manuscript. All authors read and approved the final manuscript.

\section{Funding}

This work was supported by The Research Team of Precise Diagnosis and Treatment for Gynecologic Malignant Tumors (SZSM201812041).

\section{Availability of data and materials}

Not applicable.

Ethics approval and consent to participate

Not applicable.

\section{Consent for publication}

Not applicable.

\section{Competing interests}

On behalf of all authors, the corresponding author states that there are no competing interests.

\section{Author details}

'Department of Gynecology, The First Affiliated Hospital of Shenzhen University, Health Science Center, Shenzhen Second People's Hospital, 3002 Sungang West Road, Futian District, Shenzhen 518000, Guangdong province, China. ${ }^{2}$ Key Laboratory of Anti-Inflammatory and Immune Medicine, Ministry of Education, Anhui Collaborative Innovation Center of Anti-Inflammatory and Immune Medicine, Institute of Clinical Pharmacology, Anhui Medical University, 81 Meishan Road, Hefei 230032, Anhui province, China.

Received: 26 December 2019 Accepted: 18 May 2020

Published online: 05 June 2020

\section{References}

1. Hummitzsch $\mathrm{K}$, Anderson RA, Wilhelm D, Wu J, Telfer EE, Russell DL, et al. Stem cells, progenitor cells, and lineage decisions in the ovary. Endocr Rev. 2015:36:65-91.

2. Thomas FH, Vanderhyden BC. Oocyte-granulosa cell interactions during mouse follicular development: regulation of kit ligand expression and its role in oocyte growth. Reprod Biol Endocrinol. 2006;4:1-8.

3. Aerts JMJ, Bols PEJ. Ovarian follicular dynamics: a review with emphasis on the bovine species. Part l: Folliculogenesis and pre-antral follicle development. Reprod Domest Anim. 2010;45:171-9.

4. Yilmaz B, Vellanki P, Ata B, Yildiz BO. Metabolic syndrome, hypertension, and hyperlipidemia in mothers, fathers, sisters, and brothers of women with polycystic ovary syndrome: A systematic review and meta-analysis. Fertil Steril [Internet]. Elsevier Inc. 2018; Available from:. https://doi.org/10.1016/j. fertnstert.2017.10.018.

5. Collins G, Patel B, Thakore S, Liu J. Primary Ovarian Insufficiency: Current Concepts. South Med J [Internet]. 2017;110:147-53. Available from: http:// sma.org/southern-medical-journal/article/primary-ovarian-insufficiencycurrent-concepts.

6. Pfeifer S, Butts S, Dumesic D, Fossum G, Gracia C, La Barbera A, et al. Prevention and treatment of moderate and severe ovarian hyperstimulation syndrome: a guideline. Fertil Steril. 2016;106:1634-47.

7. Färkkilä A, Haltia U-M, Tapper J, McConechy MK, Huntsman DG, Heikinheimo M. Pathogenesis and treatment of adult-type granulosa cell tumor of the ovary. Ann Med [Internet]. 2017:49:435-47 Available from: https://www.tandfonline.com/doi/full/10.1080/07853890.2017.1294760.

8. Escobar-Morreale HF, Millán JLS. Abdominal adiposity and the polycystic ovary syndrome. Trends Endocrinol Metab. 2007;18:266-72.

9. Baber R. Primary ovarian insufficiency. Med Today. 2014;15:73-5.

10. Grossman LC, Michalakis KG, Browne H, Payson MD, Segars JH. The pathophysiology of ovarian hyperstimulation syndrome: an unrecognized compartment syndrome. Fertil Steril [internet]. Elsevier Ltd. 2010;94:1392-8. Available from:. https://doi.org/10.1016/j.fertnstert.2009.07.1662.

11. Cheng W-T, Rosario R, Muthukaruppan A, Wilson MK, Payne K, Fong PC, et al. MicroRNA profiling of ovarian granulosa cell tumours reveals novel diagnostic and prognostic markers. Clin Epigenetics. 2017;9:72 Available from: http://clinicalepigeneticsjournal.biomedcentral.com/articles/10.1186/ s13148-017-0372-0. 
12. Wang $Y$, Navin NE. Advances and applications of single cell sequencing technologies. Mol Celll. 2015;58:598-609.

13. Liao J, Wang J, Liu Y, Li J, Duan L. Transcriptome sequencing of InCRNA, miRNA, mRNA and interaction network constructing in coronary heart disease. BMC Med Genomics. 2019;12:1-12.

14. Li CQ, Huang GW, Wu ZY, Xu YJ, Li XC, Xue YJ, et al. Integrative analyses of transcriptome sequencing identify novel functional IncRNAs in esophageal squamous cell carcinoma. Oncogenesis. 2017;6:1-14.

15. M.W. W, E.A. B. Naming "junk": human non-protein coding RNA (ncRNA) gene nomenclature. Hum Genomics [Internet]. 2011;5:90-8. Available from: http://www.embase.com/search/results?subaction=viewrecord\&from= export\&id=L361734383.

16. Kitagawa M, Kotake Y, Ohhata T. Long Non-Coding RNAs Involved in Cancer Development and Cell Fate Determination. Curr Drug Targets [Internet]. 2012; 13:1616-21. Available from: http://www.eurekaselect.com/openurl/content. php?genre $=$ article\&issn $=1389-4501$ \& volume $=13 \&$ issue $=13 \&$ spage $=1616$.

17. Ma L, Li A, Zou D, Xu X, Xia L, Yu J, et al. LncRNAWiki: harnessing community knowledge in collaborative curation of human long non-coding RNAs. Nucleic Acids Res. 2015:43:D187-92

18. Derrien T, Johnson R, Bussotti G, Tanzer A, Djebali S, Tilgner $H$, et al. The GENCODE v7 catalog of human long noncoding RNAs: analysis of their gene structure, evolution, and expression. Genome Res. 2012;22:1775-89.

19. Ørom UA, Derrien T, Beringer M, Gumireddy K, Gardini A, Bussotti G, et al. Long noncoding RNAs with enhancer-like function in human cells. Cell. 2010;143:46-58. Available from:. https://doi.org/10.1016/j.cell.2010.09.001.

20. Heintzman ND, Stuart RK, Hon G, Fu Y, Ching CW, Hawkins RD, et al. Distinct and predictive chromatin signatures of transcriptional promoters and enhancers in the human genome. Nat Genet. 2007;39:311-8.

21. Ledford H. Circular RNAs throw genetics for a loop. Nature. 2013;494:415.

22. Akrami R, Jacobsen A, Hoell J, Schultz N, Sander C, Larsson E. Comprehensive analysis of Long non-coding RNAs in ovarian Cancer reveals global patterns and targeted DNA amplification. PLoS One. 2013;8:1-11.

23. Xiong Y, Liu T, Wang S, Chi H, Chen C, Zheng J. Cyclophosphamide promotes the proliferation inhibition of mouse ovarian granulosa cells and premature ovarian failure by activating the IncRNA-Meg3-p53-p66Shc pathway. Gene. 2017:596:1-8. Available from:. https://doi.org/10.1016/j.gene.2016.10.011.

24. Li J, Cao Y, Xu X, Xiang H, Zhang Z, Chen B, et al. Increased new IncRNAmRNA gene pair levels in human cumulus cells correlate with oocyte maturation and embryo development. Reprod Sci. 2015;22:1008-14.

25. Zhao J, Huang J, Geng X, Chu W, Li S, Chen Z-J, et al. Polycystic ovary syndrome: novel and hub IncRNAs in the insulin resistance-associated IncRNA-mRNA network. Front Genet. 2019:10:1-12.

26. Tripathi MK, Doxtater K, Keramatnia F, Zacheaus C, Yallapu MM, Jaggi M, et al. Role of IncRNAs in ovarian cancer: defining new biomarkers for therapeutic purposes. Drug Discov Today. 2018;23:1635-43. Available from:. https://doi.org/10.1016/j.drudis.2018.04.010.

27. Wang $X-Y$, Qin $Y-Y$. Long non-coding RNAs in biology and female reproductive disorders. Fontiers Biosci. 2019;24:750-64.

28. Sánchez F, Smitz J. Molecular control of oogenesis. Biochim Biophys Acta Mol Basis Dis. 2012;1822:1896-912. Available from:. https://doi.org/10.1016/j. bbadis.2012.05.013.

29. Yerushalmi GM, Salmon-Divon M, Yung Y, Maman E, Kedem A, Ophir L, et al. Characterization of the human cumulus cell transcriptome during final follicular maturation and ovulation. Mol Hum Reprod. 2014;20:719-35.

30. Xu X-F, Li J, Cao Y-X, Chen D-W, Zhang Z-G, He X-J, et al. Differential Expression of Long Noncoding RNAs in Human Cumulus Cells Related to Embryo Developmental Potential. Reprod Sci [Internet]. 2015;22:672-8. Available from: http://journals.sagepub.com/doi/10.1177/1933719114561562.

31. Macaulay AD, Gilbert I, Caballero J, Barreto R, Fournier E, Tossou P, et al. The Gametic synapse: RNA transfer to the bovine oocyte. Biol Reprod [Internet]. 2014;91:1-12 Available from: https://academic.oup.com/biolreprod/articlelookup/doi/10.1095/biolreprod.114.119867.

32. Yan L, Yang M, Guo H, Yang L, Wu J, Li R, et al. Single-cell RNA-Seq profiling of human preimplantation embryos and embryonic stem cells. Nat Struct Mol Biol. 2013;20:1131-9.

33. Xue Z, Huang K, Cai C, Cai L, Jiang CY, Feng Y, et al. Genetic programs in human and mouse early embryos revealed by single-cell RNA sequencing. Nature. 2013:500:593-7.

34. Bouckenheimer J, Fauque P, Lecellier CH, Bruno C, Commes T, Lemaître JM, et al. Differential long non-coding RNA expression profiles in human oocytes and cumulus cells. Sci Rep. 2018:8:1-13.
35. Han Q, Zhang W, Meng J, Ma L, Li A. LncRNA-LET inhibits cell viability, migration and EMT while induces apoptosis by up-regulation of TIMP2 in human granulosa-like tumor cell line KGN. Biomed Pharmacother. 2018;100: 250-6.

36. Allard S, Adin P, Gouédard L, di Clemente N, Josso N, Orgebin-Crist MC, et al. Molecular mechanisms of hormone-mediated Müllerian duct regression: involvement of beta-catenin. Development. 2000;127:3349-60 Available from: http://www.ncbi.nlm.nih.gov/pubmed/10887090.

37. Kimura AP, Yoneda R, Kurihara M, Mayama S, Matsubara S. A long noncoding RNA, IncRNA-Amhr2, plays a role in Amhr2 gene activation in mouse ovarian granulosa cells. Endocrinology. 2017;158:4105-21.

38. Xu P, Bao R, Zhang Y, Lu E, Feng F, Zhang L, et al. Prolyl oligopeptidase regulates progesterone secretion via the ERK signaling pathway in murine luteal cells. Mol Reprod Dev. 2019;86:714-26.

39. Matsubara S, Kurihara M, Kimura AP. A long non-coding RNA transcribed from conserved non-coding sequences contributes to the mouse prolyl oligopeptidase gene activation. J Biochem. 2014;155:243-56.

40. Yung Y, Ophir L, Yerushalmi GM, Baum M, Hourvitz A, Maman E. HAS2-AS1 is a novel LH/hCG target gene regulating HAS2 expression and enhancing cumulus cells migration. J Ovarian Res. 2019;12:1-7.

41. Liu Y, Li Y, Feng S, Ye D, Chen $X$, Zhou $X$, et al. Long Noncoding RNAs: Potential Regulators Involved in the Pathogenesis of Polycystic Ovary Syndrome. Endocrinology [Internet]. 2017;158:3890-9. Available from: http:// academic.oup.com/endo/article/158/11/3890/4100163.

42. Huang $X$, Hao C, Bao H, Wang M, Dai H. Aberrant expression of long noncoding RNAs in cumulus cells isolated from PCOS patients. J Assist Reprod Genet. 2016;33:111-21.

43. Legro RS, Arslanian SA, Ehrmann DA, Hoeger KM, Murad MH, Pasquali R, et al. Diagnosis and treatment of polycystic ovary syndrome: an endocrine society clinical practice guideline. J Clin Endocrinol Metab. 2013;98:4565-92.

44. Gleicher N, Weghofer A, Barad DH. The role of androgens in follicle maturation and ovulation induction: friend or foe of infertility treatment? Reprod Biol Endocrinol. 2011;9:1-12.

45. Jin L, Yang Q, Zhou C, Liu L, Wang H, Hou M, et al. Profiles for long noncoding RNAs in ovarian granulosa cells from women with PCOS with or without hyperandrogenism. Reprod BioMed Online. 2018;37:613-23.

46. Li Y, Wang H, Zhou D, Shuang T, Zhao H, Chen B. Up-regulation of Long noncoding RNA SRA promotes cell growth, inhibits cell apoptosis, and induces secretion of estradiol and progesterone in ovarian granular cells of mice. Med Sci Monit. 2018:24:2384-90.

47. Li Y, Zhao W, Wang H, Chen C, Zhou D, Li S, et al. Silencing of LncRNA steroid receptor RNA activator attenuates polycystic ovary syndrome in mice. Biochimie. 2019;157:48-56.

48. Huang $X$, Pan J, Wu B, Teng X. Construction and analysis of a IncRNA (PWRN2)-mediated ceRNA network reveal its potential roles in oocyte nuclear maturation of patients with PCOS. Reprod Biol Endocrinol. 2018; $16: 1-13$.

49. Li R, Zhang L, Jia L, Duan Y, Li Y, Bao L, et al. Long non-coding RNA BANCR promotes proliferation in malignant melanoma by regulating MAPK pathway activation. PLoS One. 2014;9:1-9.

50. Wang D, Wang D, Wang N, Long Z, Ren X. Long non-coding RNA BANCR promotes endometrial Cancer cell proliferation and invasion by regulating MMP2 and MMP1 via ERK/MAPK signaling pathway. Cell Physiol Biochem. 2016:40:644-56

51. Yang R, Chen J, Wang L, Deng A. LncRNA BANCR participates in polycystic ovary syndrome by promoting cell apoptosis. Mol Med Rep. 2019;19:1581-6.

52. Zhao J, Xu J, Wang W, Zhao H, Liu H, Liu X, et al. Long non-coding RNA LINC-01572:28 inhibits granulosa cell growth via a decrease in p27 (Kip1) degradation in patients with polycystic ovary syndrome. EBioMedicine The Authors. 2018;36:526-38.

53. Che Q, Liu M, Zhang D, Lu Y, Xu J, Lu X, et al. Long noncoding RNA HUPCOS promotes follicular fluid androgen excess in PCOS patients via aromatase inhibition. J Clin Endocrinol Metab. 2020;105:1-12.

54. Liu G, Liu S, Xing G, Wang F. IncRNA PVT1/MicroRNA-17-5p/PTEN Axis regulates secretion of $E 2$ and $P 4$, proliferation, and apoptosis of ovarian Granulosa cells in PCOS. Mol Ther - Nucleic Acids. 2020;20:205-16.

55. Ying L, Shi-ling C. Upregulation of the long non-coding RNA TUG1 inhibits granulosa cell apoptosis and autophagy in polycystic ovary syndrome by regulating ERK/MAPK pathway. Fertil Steril. 2019;ASEM Abstr:245-6.

56. Hussein MR. Apoptosis in the ovary: molecular mechanisms. Hum Reprod Update. 2005;11:161-77. 
57. Wang X, Zhang X, Dang Y, Li D, Lu G, Chan W-Y, et al. Long noncoding RNA HCP5 participates in premature ovarian insufficiency by transcriptionally regulating MSH5 and DNA damage repair via YB1. Nucleic Acids Res. 2020;1:1-12.

58. Elizur SE, Dratviman-Storobinsky O, Derech-Haim S, Lebovitz O, Dor J, Orvieto $R$, et al. FMR6 may play a role in the pathogenesis of fragile $X$ associated premature ovarian insufficiency. Gynecol Endocrinol. 2016;32: 334-7.

59. Lin H, Li Y, Xing W, Qiu Q, Wang W, Zhang Q. Genome-wide screening differential long non-coding RNAs expression profiles discloses its roles involved in OHSS development. J Assist Reprod Genet. 2018;2:1473-82.

60. Petroff BK, Roby KF, Gao X, Son DS, Williams S, Johnson D, et al. A review of mechanisms controlling ovulation with implications for the anovulatory effects of polychlorinated dibenzo-p-dioxins in rodents. Toxicology. 2001; 158:91-107.

61. Mandal PK. Dioxin: a review of its environmental effects and its aryl hydrocarbon receptor biology. J Comp Physiol B Biochem Syst Environ Physiol. 2005;175:221-30

62. Ruszkowska M, Nynca A, Paukszto L, Sadowska A, Swigonska S, Orlowska K, et al. Identification and characterization of long non-coding RNAs in porcine granulosa cells exposed to 2,3,7,8-tetrachlorodibenzo-p-dioxin. J Anim Sci Biotechnol. 2018:9:1-13.

63. Ma XY, Wang JH, Wang JL, Ma CX, Wang XC, Liu FS. Malat1 as an evolutionarily conserved IncRNA, plays a positive role in regulating proliferation and maintaining undifferentiated status of early-stage hematopoietic cells. BMC Genomics. 2015;16:1-11. Available from:. https:/ doi.org/10.1186/s12864-015-1881-X.

64. Ji P, Diederichs S, Wang W, Böing S, Metzger R, Schneider PM, et al. MALAT1 , a novel noncoding RNA, and thymosin $\beta 4$ predict metastasis and survival in early-stage non-small cell lung cancer. Oncogene. 2003;22:8031-41.

65. Gutschner T, Hämmerle M, Diederichs S. MALAT1 - a paradigm for long noncoding RNA function in cancer. J Mol Med. 2013;91:791-801.

66. Li Y, Liu YD, Chen SL, Chen X, Ye DS, Zhou XY, et al. Down-regulation of long non-coding RNA MALAT1 inhibits granulosa cell proliferation in endometriosis by up-regulating P21 via activation of the ERK/MAPK pathway. Mol Hum Reprod. 2018;25:17-29.

\section{Publisher's Note}

Springer Nature remains neutral with regard to jurisdictional claims in published maps and institutional affiliations.

Ready to submit your research? Choose BMC and benefit from:

- fast, convenient online submission

- thorough peer review by experienced researchers in your field

- rapid publication on acceptance

- support for research data, including large and complex data types

- gold Open Access which fosters wider collaboration and increased citations

- maximum visibility for your research: over $100 \mathrm{M}$ website views per year

At $\mathrm{BMC}$, research is always in progress.

Learn more biomedcentral.com/submissions 